graphically remote cultures as communications improved over the centuries, it may be that we can extract one conclusion from Simonton's elaborate computation: those whose works have survived longest, and those whose works are most often performed, shape the "norms" in musical composition!

Sophisticated mathematical models may be misleading in another way - they may provide precise descriptions of numerical data which are quite implausible in terms of real-world processes. Simonton elegantly reviews data on changes in human creativity in politics, arts and sciences between the ages of 20 and 70 . Alas, though cheerful exceptions abound, the unmistakeable trend is for peaks to be reached between 35 and 45 with an accelerated decline thereafter. He neatly fits an algebraic model to the data (pp.110 and 111), and on p. 109 describes the functional implications of this model:

Each creator uses up [sic] this supply of creative potential [determined individually - perhaps at birth?] during his or her productive career by transforming potential into contributions.

I wish that I could believe that, by prudent inertia, I may yet reserve some "creative potential", undissipated by book reviews and unclouded by time, to astound my clapped-out contemporaries when I reach my 80s.

Incidentally it is encouraging for common sense to note that Simonton's algebraic statement of his model does not actually have this, or any other, clear functional implication. It seems to be a neutral equation for fitting empirical data using one invariant constant (e), two other constants empirically derived for particular disciplines $(a$ and $b$ ) and a fourth constant empirically derived for individuals $(c)$. It is not a parsimonious equation.

Simonton is clearly a very hard-working academic, and seems sincerely anxious that his subject should help us to understand ourselves better, to learn to anticipate and to avoid political violence, and to give each other just credit for real achievements. His book will be marvellous bed-time reading for any academic with a curiosity about historical or statistical matters. Only part of its entertainment value lies in the detection of charming errors and non sequiturs. Simonton has an acute sense of really interesting, though possibly insoluble, problems, and whenever he seems to go wrong he sets us the important task of wondering whether, and precisely how, something useful may yet be said. In spite (surely?) of the most extreme temptations he brings to his material no detectable humour. But, for the reader with his own sense of fun, this will be a delightful book.

Patrick Rabbitt is Professor of Gerontology and Cognitive Psychology at the University of Manchester.

\section{Cue for cooperation}

\section{F.H. Bronson}

Mammalian Semiochemistry: The

Investigation of Chemical Signals

Between Mammals.

By Eric S. Albone.

Wiley: 1984. Pp. 360. £29.50, \$57.

MosT mammals are nocturnal and by human standards they are quite small. Thus they rely heavily upon their chemical senses when interacting with other members of their own species, avoiding predators or searching for food. Chemical cues play many crucial roles even in mammals that are larger, more diurnal, and hence more visually-orientated. The term "semiochemical" encompasses all such cues, whether they originate from plants or animals, and incorporates the concept of communication via pheromones.

By its very nature, the broad study of chemical cueing requires close collaboration between the chemist and the biologist; as witnessed by its impact on entomology, the result can be immensely rewarding. Research on mammals, however, has thus far been dominated by behaviourists and physiologists interested strictly in communication between members of the same species. Dr Albone is one of the few chemists whose primary interest is in mammalian chemical cueing, and this is the first account of the subject written by a chemist. The strengths and weaknesses of the book are those that might be expected when a scientist of one discipline looks at a complex subject that has its roots in another, markedly different discipline.

The first two chapters of the book introduce the subject and note the potential contributions and limitations of chemical investigations; of particular interest here is the author's emphasis on the

\section{SOM introduction}

\section{P.W. Hawkes}

Theory and Practice of Scanning Optical Microscopy.

By Tony Wilson and Colin Sheppard. Academic: 1984. Pp.213. £25, \$39.50.

THERE are not many types of radiation for which high-quality, two-dimensional, intensity-sensitive recording media exist. Light and electrons are exceptional in this respect, so that (apart from a few early scanning electron instruments) scanning microscopes were developed long after their fixed-beam counterparts had become commonplace. It is, however, gradually being recognized that the optics of scanning systems and the sequential nature of the image-forming process possess probable complexity of most mammalian chemical cues. The next six chapters deal with the potential sources of the cues and the types of compounds that may arise from these sources. Thus the skin, scent glands, urine, breath and the reproductive tract are considered in detail. Unfortunately much of this discussion relies upon data collected in human beings, and in only a few cases are sources and compounds related directly to functions (this, it should be said, is not the fault of the author, but an accurate reflection of the state of our knowledge). In a further chapter that should be read by everyone interested in the topic, Dr Albone considers the important role played by microorganisms in shaping odours. Finally, a guest contributor, Dr Stephen Shirley, has written a nice primer on the neurophysiology of the chemical senses.

While this is a historically important book, dealing as it does with a young and rapidly developing area, it will probably fail in its stated objective of bringing together biologists and chemists. Much of what the biologist finds exciting about the subject is treated only superficially - the integration of chemical cues with information provided by the mammal's other senses; the shaping of responses by experience and context; the adaptive functions of signalling systems; and so forth. On the other hand, one can hope that the book will attract more chemists into semiochemical research, and all biologists interested in chemical cueing certainly should read it because it will help them understand how a chemist approaches many of the problems on which they work. Ultimately, real progress will occur only through cooperation, and scientists in both disciplines need to understand much better how their opposite numbers view this challenging field.

F. H. Bronson is Professor of Zoology at the University of Texas, Austin.

attractive features in their own right, with the result that commercial scanning electron microscopes have been with us for nearly 20 years and commercial scanning transmission electron microscopes for about a decade. Now, as this book convincingly demonstrates, the scanning optical microscope (SOM) is beginning to supplement its conventional ancestor.

No two people are better qualified to write a book on SOMs than Tony Wilson and Colin Sheppard, for almost all the work on the theory and performance of these instruments has come from their Department of Engineering Science in Oxford. In extremely simple language, and using mathematics that anyone familiar with Sections 9.5-10.5 of Born and Wolf's Principles of Optics will find easy to follow, they take us through the theory of image formation in the SOM. The two operating modes - essentially large 